\title{
LABORATORY ASTROPHYSICS AND ASTROCHEMISTRY IN THE HERSCHEL/ALMA ERA
}

\author{
J. Cernicharo ${ }^{1}$
}

\begin{abstract}
The interpretation of molecular line surveys at millimetre and submillimetre wavelengths towards astrophysical objects requires a good knowledge of the spectroscopy of the molecules present in the gas phase, and of the basic collisional processes between them and molecular and atomic Hydrogen, Helium, and electrons. From the chemical point of view, the modeling of these data requires a large amount of laboratory information concerning the reactivity of these species in the gas phase and in the ice mantles of dust grains. In this contribution I will focus on the problems to interpret line surveys and I will discuss how we will have to proceed in the new era opened by ALMA where sensitivity and angular resolution will surpass by nearly a factor 10 those of existing facilities, and where the spectral confusion limit could be reached towards many sources.
\end{abstract}

\section{Introduction}

The origin and evolution of the molecular universe starts with the injection of material into the interstellar medium by stars in the later stages of their evolution, followed by the processing of this material by the prevalent ultraviolet radiation fields, energetic particles, and strong shocks, and ends with the incorporation of this material into newly formed stars and their planetary systems. Understanding this chemical evolution and its relationship to the origin of life on Earth and possibly other planetary systems in the universe is a key problem in astrophysics. Over the last 30 years, some 160 molecules have been detected in space (see, e.g., Herbst \& van Dishoeck 2009). These range from simple diatomics such as molecular hydrogen and carbon monoxide, to complex species such as large carbon chains containing up to 11 carbon atoms (Cernicharo et al. 2000), cyclic species such as benzene (Cernicharo et al. 2001a,b) and fullerenes (Cami et al. 2010; Sellgren et al. 2010). The presence of Polycyclic Aromatic Hydrocarbon (PAH)

${ }^{1}$ Department of Astrophysics, CAB. INTA-CSIC, Crta Torrejón km 4, 28850 Torrejón de Ardoz, Madrid, Spain; e-mail: jcernicharo@cab.inta-csic.es 
molecules containing typically 50-100 C-atoms has also been proposed (Leger \& Puget 1984; Allamandola et al. 1985; Joblin \& Tielens 2011).

Over the next years, the new observatories coming on line (Atacama Large Millimetre Array, extremely large telescopes) will increase the existing inventory of the molecular universe by orders of magnitude. Identification of the detected lines with specific molecular species, their analysis in terms of the physical conditions in the observed regions, and implication for the physical and chemical processes ruling the universe require a close synergy between laboratory spectroscopists, molecular physicists, chemists, and astronomers (see Fig. 1). This requires a new and innovative approach to this field that is precisely addressed in this conference.

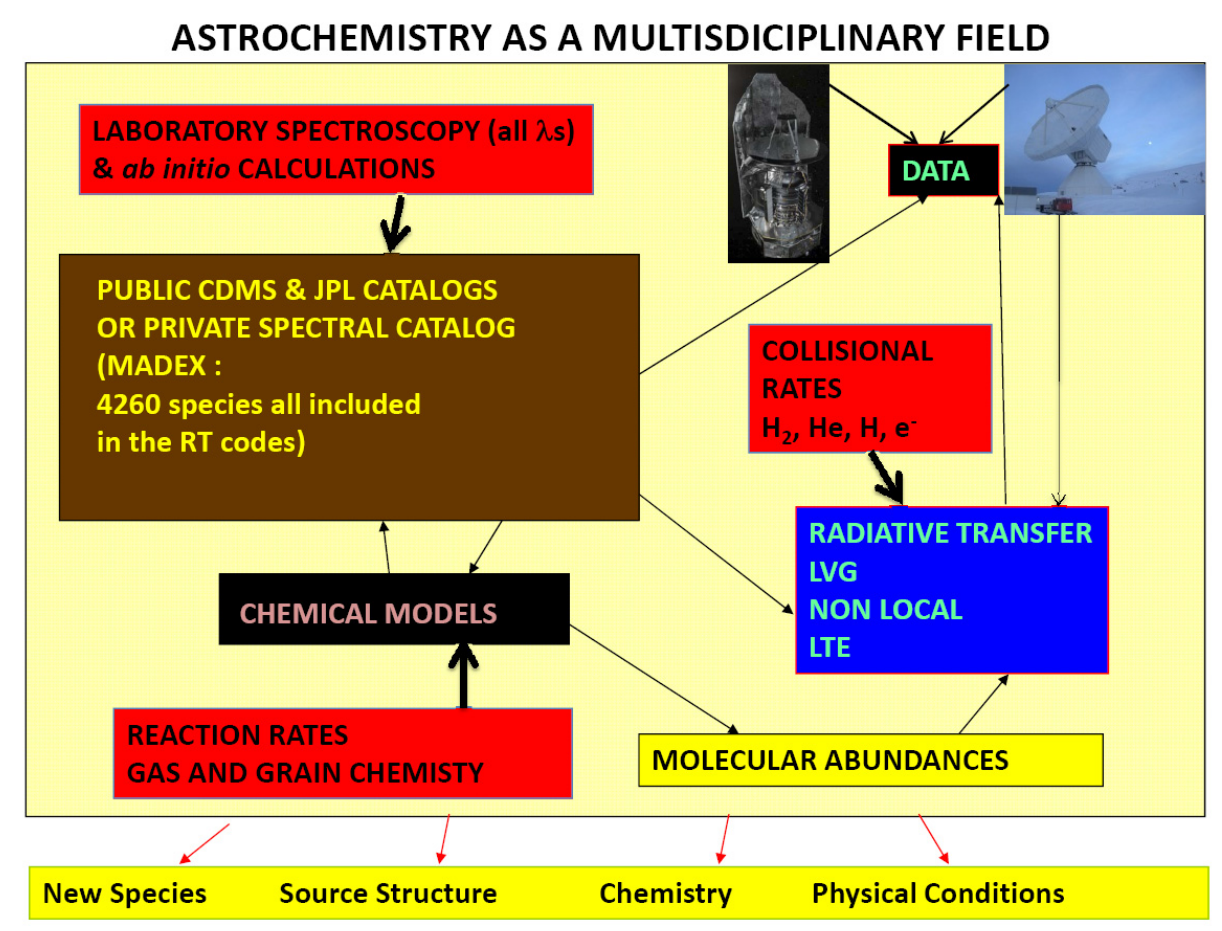

Fig. 1. The different disciplines involved in the interpretation of molecular observations in Astrophysics. The role of laboratory astrophysics, both experimental and theoretical, is indicated with thicker arrows (red boxes).

The identification of specific species in space requires direct comparison of the particular frequencies of emission or absorption lines observed in interstellar space with spectroscopic measurements of known species in a controlled laboratory experiment. In order to interpret the Molecular Astrophysics observations, supporting molecular physics quantum chemical calculations are required:

I) The intensities of the rotational lines observed in space through radio astronomical techniques depend, when they are optically thin, directly on the collisional 
excitation rates of the molecules with the predominant collision partners, $\mathrm{H}, \mathrm{H}_{2}$, $\mathrm{He}, \mathrm{H}^{+}, \mathrm{e}^{-}$. These rates will have to be calculated using quantum chemical methods or measured in the laboratory by molecular physicists.

II) The abundances of interstellar molecules are the result of a balance between formation and destruction reactions. The rate coefficients and products of relevant reactions will have to be measured under astrophysically relevant conditions (e.g., low temperature, low pressure) or quantum chemically calculated. These rates will then be used by astronomers in models to calculate the abundances of interstellar species. Gas phase and dust grain chemical processes have to be studied in the laboratories.

III) Laboratory spectroscopists need to measure the transition frequencies and dipole moments of astrophysically relevant molecules.

IV) All of these data together can then be used by astronomical modelers to predict the expected line intensities of new species which can then be targeted in specific searches and to derive the physical conditions of the observed sources.

It is clear that action in these scientific areas has to be strongly interwoven in studies of the molecular universe (see Fig. 1). In the last years several key topics in the areas of molecular complexity in space and in the chemistry of regions of star and planet formation have been identified: water in the universe, carbon chemistry, deuterium, chemical complexity, ionization and photodissociation, oxygen and nitrogen chemistry, molecular tracers of shocks, and interstellar/circumstellar dust grain chemistry.

In this contribution I will focus on the interpretation of line surveys and the problems we will need to confront to get the maximum return from extremely sensitive astronomical instruments such as ALMA that will start soon to provide new and spectacular data.

\section{Line surveys and chemical complexity}

The last 20 years have represented a real burst in the field of Molecular Astrophysics thanks to the availability to the community of very large ground-based radio and infrared telescopes (IRAM, ESO instruments, JCMT, CSO), and European and US Space far-IR spectroscopic missions like the Infrared Space Observatory (ISO) and the Spitzer Space Telescope. The new generation of telescopes, like the Herschel Space Observatory of the European Space Agency successfully launched on May 2009 (PIlbratt et al. 2010), or the ALMA project, are providing the astrophysical community with instruments having 10 (at millimetre/submillimetre wavelengths) - 40 (at far-infrared wavelengths) times more collecting area and angular resolution, than those currently available. These performances will allow studying the chemical evolution of the Universe from 800-1000 Myr after the big-bang up to now, and will raise new and exciting problems concerning the formation of planets and the prebiotic chemistry leading to the origin of life. In particular, Herschel has opened the opportunity to study the role of water vapour in the physical and chemical evolution of molecular clouds and protoplanetary disks (van Dishoeck et al. 2011). The interpretation of these observations requires 
the characterization of a huge amount of molecular properties in order to obtain the physical and chemical conditions of the molecular gas across the Universe. Molecules have an important influence on the heating and cooling of interstellar gas and, therefore, on the cloud structure of the interstellar medium in galaxies. Characterizing the origin and evolution of interstellar molecules is therefore a fundamental goal of modern astrophysics and a key issue in understanding the chemical evolution of the Universe and its implication on the origin of life. As previously commented, a variety of observations have shown that surprisingly complex molecules are an important component of the interstellar medium. These include the vibrational signatures of large PAH molecules, the electronic fingerprints of a variety of complex molecules that are the carriers of the diffuse absorption bands in the visible spectra of stars (Herbig 1995), and the rotational transitions of some 160 smaller molecules most of them discovered in the microwave, millimetre, submillimetre and far-infrared spectral ranges. Some key molecules, such as acetylene and longer polyacetylenic chains, lack of permanent dipole moment and have to be observed in the mid- and near-infrared domains (Cernicharo et al. 2001a,b; Fonfría et al. 2008, 2011).

The most powerful tool to study the chemical complexity of interstellar and circumstellar clouds is the systematic observation of a source at all wavelengths (see, e.g., Bergin et al. 2010). Line surveys allow to produce a complete census of the molecular content of these sources. The new receivers and spectrometers installed in most radio telescopes allow to perform such studies in a reasonable amount of time, say a couple of days compared with the several weeks, often with observing runs spreading over the years, that were needed in the 80's and 90's of the last century (see, e.g., Cernicharo et al. 2000). Line surveys allow to observe many lines of the same molecule and its isotopologues permitting the determination of the physical and chemical structure of molecular clouds. An important aspect to take into account when looking for chemical complexity is the frequency coverage. While the high frequencies provided by the HIFI instrument on board Herschel are ideal to trace the chemical and physical conditions of warm gas through the observation of light species (see Fig. 2 and Bergin et al. 2010), millimetre and submillimetre frequencies are better adapted for polyatomic molecules having less than 8-10 atoms. Heavier molecules can be studied at microwave wavelengths and for that purpose the VLA, the low frequency bands of ALMA and the future Square Kilometer Array (SKA) will play a key role in progressing in the study of chemical complexity in molecular and circumstellar clouds.

\subsection{How to deal with the spectral confusion limit in Astrophysics}

The Orion KL (Kleinmann-Low) cloud is the prototype and best studied case of high mass star forming region in our Galaxy. At a distance of 414 pc (Menten et al. 2007; see Genzel \& Stutzki 1989 for review of the properties of this source), it is the nearest region of high mass star formation. The chemistry in this cloud is particularly rich as a result of the interaction of the newly formed stars and their 

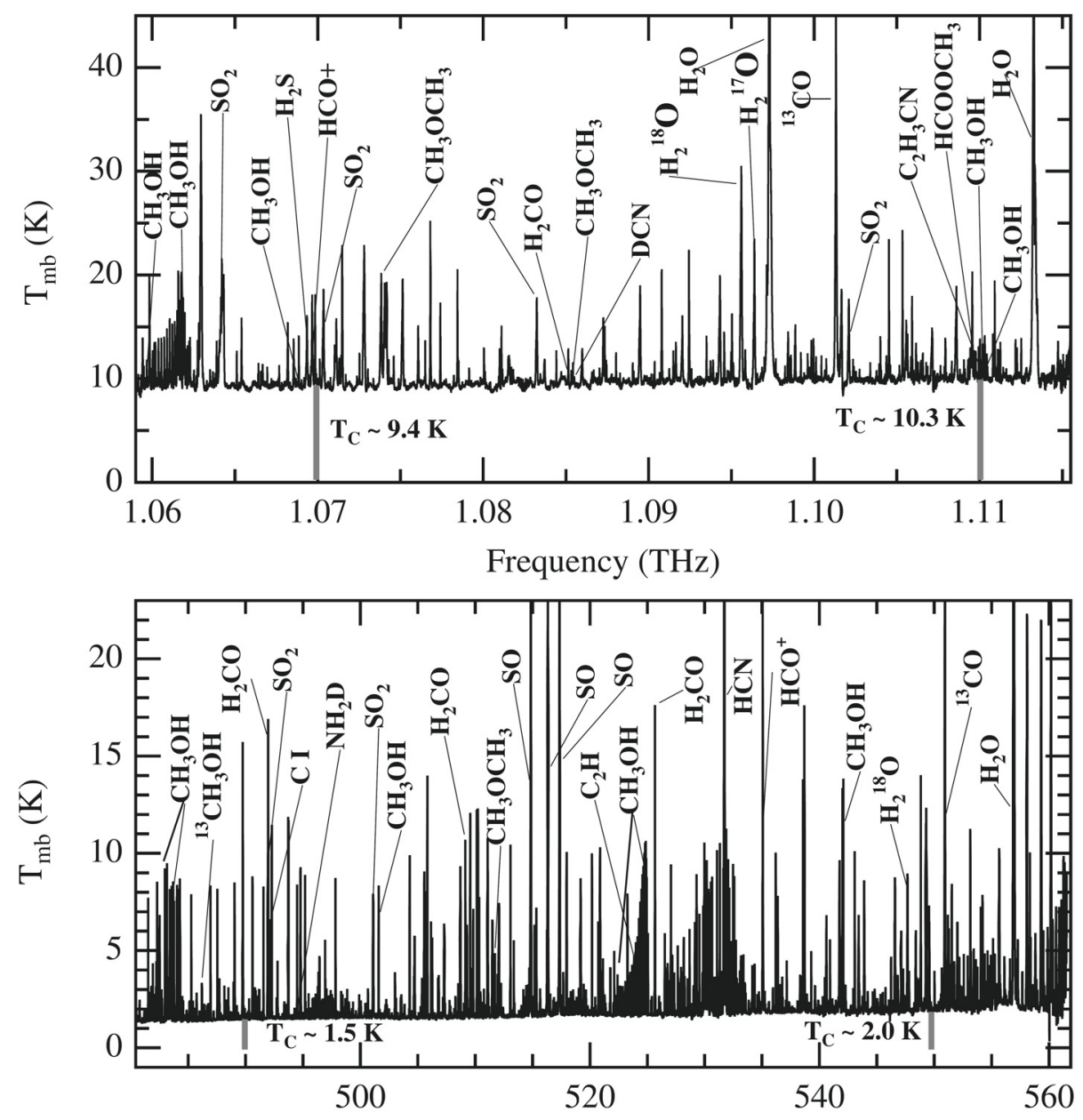

Fig. 2. Top: HIFI spectral scan of Orion KL in Band 4b. Bottom: HIFI spectral scan of Orion KL in Band 1a. Strong lines in both spectra are identified (from Bergin et al. 2010).

environment. The evaporation of dust mantles in some regions of the KL cloud and the high kinetic temperature of the gas produce a wide variety of molecules in the gas phase that are responsible for a spectacularly prolific and intense line spectrum (Blake et al. 1987; Brown et al. 1988; Charnley 1997) revealed by many line surveys performed in this region in the last 24 years at different frequency ranges: 72.2-91.1 GHz by Johansson et al. (1984); 215-247 GHz by Sutton et al. (1985); 247-263 GHz by Blake et al. (1986); 200.7-202.3, 203.7-205.3 and 330-360 GHz by Jewell et al. (1989); 70-115 GHz by Turner (1989); $257-273 \mathrm{GHz}$ by Greaves \& White (1991); 150-160 GHz by Ziurys \& McGonagle (1993); 325-360 GHz by 


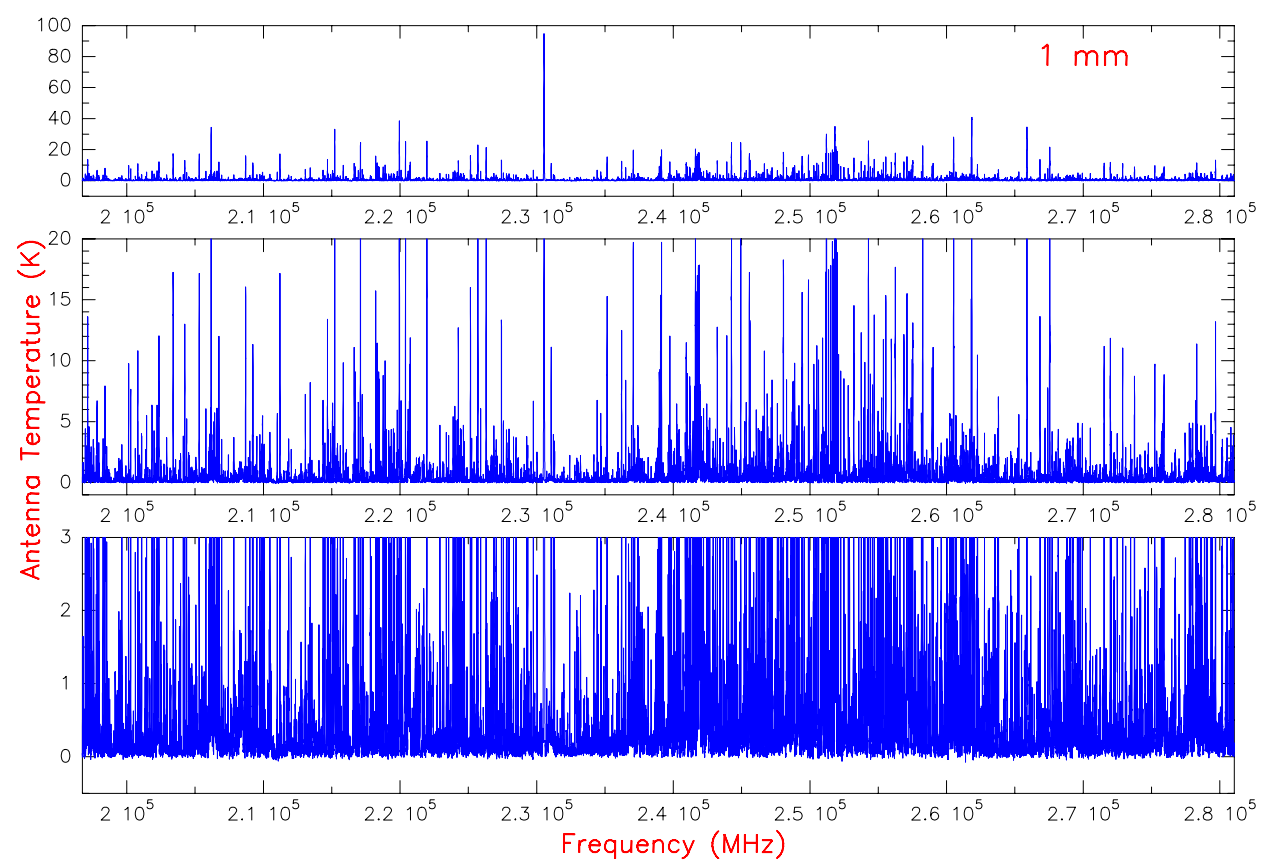

Fig. 3. The $30 \mathrm{~m}$ IRAM radio telescope line survey of Orion KL at $1 \mathrm{~mm}$ of wavelength. From Tercero et al. $(2010,2011,2012)$.

Schilke et al. (1997); 607-725 GHz by Schilke et al. (2001); 138-150 GHz by Lee et al. (2001); 159.7-164.7 GHz by Lee \& Cho (2002); 455-507 GHz by White et al. (2003); 795-903 GHz by Comito et al. (2005); 44-188 $\mu \mathrm{m}$ by Lerate et al. (2008); and the recent 500-1900 GHz line survey with the HIFI instrument from the HEXOS Key Program of Herschel (Bergin et al. 2010).

In spite of these large amounts of data, no line confusion limited surveys had been carried out with large single dish telescopes up to 2006 when Tercero \& coworkers started a line survey with the IRAM 30-m telescope at all frequencies allowed by the telescope receivers (a total frequency coverage of $\simeq 168 \mathrm{GHz}$; Tercero et al. 2010, 2011, 2012; see Figs. 3 and 4). The main goal of this line survey was to provide a deep insight into the chemistry of the Orion KL region and to refine our knowledge of its physical parameters. The final goal was to search for new molecular species. However, the number of spectral features was so large (see Figs. 3 and 4 ) that the analysis of the data was strongly handicapped by the limited coverage of spectral databases. Moreover, as shown in Figures 3 and 4 the density of weak features $\left(\mathrm{T}_{A}<0.2,0.1\right.$, and $0.05 \mathrm{~K}$ at 1,2 and $3 \mathrm{~mm}$ respectively) avoids any reasonable assignment without a detailed modeling of each molecular species, its isotopologues and its vibrational excited states. Kinetic temperatures in several of the Orion KL cloud components are often larger than $200 \mathrm{~K}$. Hence, vibrational levels of abundant species with energies below a few hundreds Kelvin 


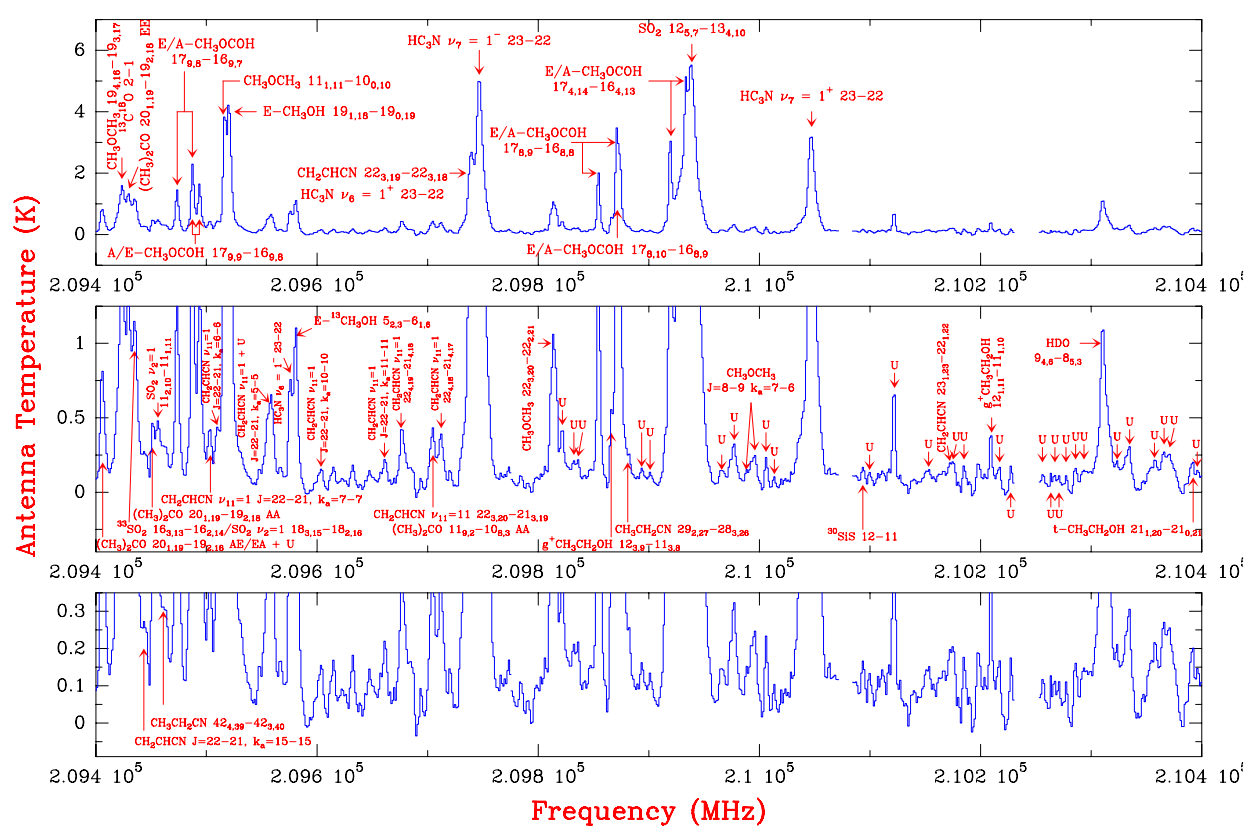

Fig. 4. A zoom to the line survey shown in Figure 2 from Tercero et al. (2010, 2011, 2012).

will be populated enough to produce significant emission. With the limits quoted above for the spectral confusion limit we had initially more than 14000 spectral features of which 8000 were unidentified. The two molecular databases with public access, CDMS (Müller et al. 2001, 2005, http://www.cdms.de) and JPL (Pickett et al. 1998, http://spec.jpl.nasa.gov), and the MADEX code (see below) were used for line assignment. In order to progress in the identification of the lines, which is a mandatory step before new species can be assigned within the forest of lines, we started a collaboration with spectroscopiscts to study the isotopologues of $\mathrm{CH}_{3} \mathrm{CH}_{2} \mathrm{CN}$ and $\mathrm{CH}_{3} \mathrm{OCOH}$ (see, Margulès et al. 2009, 2010; Demyk et al. 2007; Tercero et al. 2012). The isotopologues of these two species account for more than 800 spectral features and when their vibrationally excited states are included more than 2000 features can be assigned.

The example shown above is a special one with the present facilities. However, it will become the standard spectroscopic output of instruments such as ALMA for many sources. Although not shown in this contribution, the spectrum of evolved stars will also present forests of lines that will require specific spectroscopic work in the laboratory in order to identify molecular emission from these circumstellar envelopes. New molecules will be discovered in interstellar and circumstellar envelopes but only after the frequencies of the isotopologues and vibrationally excited states of the most abundant species have been removed, which depends on laboratory data. The synergy between Astrophysics and Molecular Spectroscopy 
has to be further developed to get the maximum scientific return of these costly new astronomical facilities. New methods have to be adopted by astronomers to look for the chemical complexity and evolution of interstellar and circumstellar clouds. In the following section I describe the tool and the method we use in our group to deal with line surveys at the spectral confusion limit.

\subsection{MADEX}

The Madrid Excitation Code (MADEX) has been developed during the last 30 years. By April 2012 it contains information on the rotational transitions and line strengths for 919 different species with a total of 4109 spectroscopic entries corresponding to the isotopologues and vibrationally excited states of these molecules. The data MADEX stores are not the frequencies of the rotational transitions but the rotational constants. MADEX also contains all available collisional rate coefficients needed to solve the statistical equilibrium equations when estimating emerging intensities of rotational lines in molecular clouds. With frequencies and collisional rates it is possible to predict under the Large Velocity Gradient (LVG) approximation, or assuming Local Thermodynamic Equilibrium (LTE) if these rates are not available, the intensities of molecular lines. When collisional rates are not available the user can select collisional rates computed by MADEX using very simple assumptions.

Many papers have been written in the last years by the Madrid group of Molecular Astrophysics using MADEX, in particular the papers based on the line survey of Orion (Tercero et al. 2010, 2011, 2012; Margulés et al. 2008, 2009; Demyk et al. 2007) but also the spectral line survey of IRC+10216 at $2 \mathrm{~mm}$ (Cernicharo et al. 2000), the discovery of propylene (Marcelino et al. 2007) and fulminic acid (HCNO; Marcelino et al. 2009), molecules present in MADEX but not in the CDMS or JPL catalogues, and a large number of ISO papers related to molecular spectroscopy in the far-infrared (see, e.g., Herpin et al. 2000; Herpin et al. 2002; Goicoechea et al. 2004; Cernicharo et al. 2006). At the beginning MADEX was just a LVG code based on the formalism described by Goldreich \& Kwan (1974). The start of operations of the $30 \mathrm{~m}$ IRAM radio telescope in 1985 represented a considerable challenge for the identification of the molecular lines detected towards interstellar and circumstellar clouds. At this time I started to introduce in the code the new molecules detected every month with the $30 \mathrm{~m}$ IRAM and the $45 \mathrm{~m}$ Nobeyama telescopes $\left(\mathrm{C}_{5} \mathrm{H}, \mathrm{C}_{6} \mathrm{H}, \mathrm{CCS}, \mathrm{C}_{3} \mathrm{~S}, \mathrm{AlF}, \mathrm{AlCl}, \mathrm{KCl}, \mathrm{NaF}, \mathrm{SiC}, \ldots\right.$, see, e.g., Cernicharo et al. 2000 and references therein). MADEX was designed to deal with the Hamiltonians describing the energy levels of the molecules and to have the rotational constants published in the literature as input parameters to compute frequencies and intensities. Unfortunately, very soon it was clear that most authors were not providing enough digits for the rotational constants, that typographical errors were common in the literature prior to electronic publications introducing large inaccuracies in the predicted frequencies, and that many authors do not provided the correlation matrix needed to compute the uncertainty of the calculated frequencies. Hence, I started to write fitting routines for each specific 
molecule, gathering all observed lines in the laboratory published in the literature, and fitting these data to obtain the best set of rotational constants. The fitting programs provide the rotational constants and covariance matrix in the form of FORTRAN routines that are directly digested by MADEX. Table 1 shows a comparison of the MADEX frequency predictions for selected rotational transitions of some species with those of the CDMS and JPL databases. For each species MADEX contains the information on the bibliographic sources for the line frequencies, the dipole moments, the standard deviation of the fit and a description of the range of frequencies and quantum numbers used in the fit. Only for $6 \%$ of species available in MADEX the data are coming directly from the CDMS or JPL spectral catalogs.

Table 1. Example of MADEX frequency predictions compared to public databases.

\begin{tabular}{|c|c|c|c|c|}
\hline Molecule & Transition & MADEX & Database & Reference \\
\hline $\mathrm{CO}$ & $\mathrm{J}=5-4$ & $576267.9311(2)$ & $576267.9305(50)$ & CDMS \\
\hline $\mathrm{CO}$ & $\mathbf{J}=\mathbf{2 0 - 1 9}$ & $2299569.8627(25)$ & $2299569.842(10)$ & CDMS \\
\hline $\mathrm{CO}$ & $\mathrm{J}=40-39$ & 4564005.784(30) & $4564005.640(53)$ & CDMS \\
\hline $\mathrm{CO}$ & $\mathrm{J}=50-49$ & $5672166.88(23)$ & $5672165.67(55)$ & CDMS \\
\hline $\mathrm{HCO}^{+}$ & $\mathrm{J}=4-3$ & $356734.2246(11)$ & $356734.2230(15)$ & CDMS \\
\hline $\mathrm{HCO}^{+}$ & $\mathrm{J}=23-22$ & 2047315.5(11) & $2047315.0(10)$ & CDMS \\
\hline CS & $\mathrm{J}=10-9$ & $489750.9216(10)$ & $489750.9210(38)$ & CDMS \\
\hline $\mathrm{CS}$ & $\mathrm{J}=40-39$ & $1949392.438(84)$ & $1949392.410(78)$ & CDMS \\
\hline $\mathrm{CCS}$ & $N_{J}=17_{16}-16_{15}$ & 219142.6745(67) & $219142.6745(85)$ & CDMS \\
\hline $\mathrm{CCO}$ & $N_{J}=7_{6}-6_{5}$ & $161729.4443(35)$ & $161729.4330(80)$ & JPL \\
\hline $\mathrm{CCO}$ & $N_{J}=22_{21}-21_{20}$ & 507782.82(28) & $507782.80(22)$ & JPL \\
\hline $\mathrm{CH}_{3} \mathrm{CH}_{2} \mathrm{CN}$ & $\mathrm{JK}_{\mathrm{a}} \mathrm{K}_{\mathrm{c}}=32_{2,30}-33_{1,33}$ & 44894.583(15) & 44894.588(11) & JPL \\
\hline $\mathrm{CH}_{3} \mathrm{CH}_{2} \mathrm{CN}$ & $\mathrm{JK}_{\mathrm{a}} \mathrm{K}_{\mathrm{c}}=12_{3,10}-13_{1,13}$ & $94015.876(5)$ & 94015.901(4) & JPL \\
\hline $\mathrm{CH}_{3} \mathrm{CH}_{2} \mathrm{CN}$ & $\mathrm{JK}_{\mathrm{a}} \mathrm{K}_{\mathrm{c}}=37_{4,33^{-}}-38_{1,38}$ & $322400.259(24)$ & $322400.329(17)$ & JPL \\
\hline $\mathrm{TiO}$ & $\mathrm{J}=5-4 \Omega=2$ & 160108.271(11) & $160108.244(22)$ & CDMS \\
\hline $\mathrm{TiO}$ & $\mathrm{J}=10-9 \Omega=1$ & $316518.993(9)$ & 316518.994(40) & CDMS \\
\hline $\mathrm{NiO}$ & $N_{J}=19_{19}-18_{18}$ & $575661.654(22)$ & $575661.656(94)$ & CDMS \\
\hline $\mathrm{HC}_{5} \mathrm{~N}$ & $J=50-49 v_{11}$ e & $133337.7989(26)$ & $133337.8009(35)$ & CDMS \\
\hline $\mathrm{HC}_{5} \mathrm{~N}$ & $J=50-49 v_{11} f$ & $133453.5559(22)$ & $133453.5596(25)$ & CDMS \\
\hline
\end{tabular}

Consequently, MADEX is a standalone code, without need to connect to the web to get molecular parameters and frequencies. Once launched, MADEX accepts commands related to the spectroscopy, the collisional rates, the physical conditions of the cloud (loops in density, temperature and molecular abundance are allowed) and can generate synthetic spectra, convolved with the telescope and spectrometer instrumental responses, that can be compared with observations using any graphic package. The executable code for Windows platforms, which can be also run on other operating systems, is available upon request. A web based page for all the frequency predictions, energies, line intensities and references is under construction and will be available through the web page of the Spanish Laboratory Astrophysics consortium ASTROMOL (http://auditore.cab.inta-csic. es/consolider-ingenio-astromol/). A complete description of the MADEX code will be pusblished elsewhere (Cernicharo 2013, in preparation). 


\section{Conclusions}

The analysis of the deep line surveys obtained in the ALMA and Herschel era requires a particular effort for laboratory spectroscopists to work on isotopologues and vibrationally excited states of species already known. This task is not as stimulating as discovering new species. However, there is no other way to proceed in sources such as Orion since lines carried by new species will always be hidden in forests of lines, which arise from the main isotopologue, rare isotopologues, and vibrationally excited states of well-known and abundant species. The analysis of the astronomical data will require specific tools to deal with these forests of lines. Astronomers have to be careful with the identification of lines using spectral catalogues. The match of a few lines with observed features is not a guarantee of detection; the information on the spectroscopic constants used to derive frequencies and intensities, their errors, the range of validity of the predictions have to be considered before claiming the detection of a new molecule. The literature contains some examples of wrong assignments using much less sensitive instruments. It would be a pity that data from the costly new astronomical facilities lead to pitfalls that could be avoided. The risk of automatic procedures in producing such errors is large and the only way to guarantee results with a high degree of confidence is to maintain strong collaborations between astronomers and experimentalists.

This paper was partially supported within the programme CONSOLIDER INGENIO 2010, under grant Molecular Astrophysics: The Herschel and ALMA Era.- ASTROMOL (Ref.: CSD200900038). We also thank the Spanish MICINN for funding support through grants AYA2006-14876 and AYA2009-07304.

\section{References}

Allamandola, L.J., Tielens, A.G.G.M., \& Barker, J.R., 1985, ApJ, 290, L25

Blake, G.A., Sutton, E.C., Masson, C.R., \& Philips, T.H., 1986, ApJS, 60, 357

Blake, G.A., Sutton, E.C., Masson, C.R., \& Philips, T.H., 1987, ApJ, 315, 621

Bergin, E.A., et al., 2010, A\&A, 521, L20

Brown, P.D., Charnley, S.B., \& Millar, T.J., 1988, MNRAS, 231, 409

Cami, J., Bernard-Salas, J., Peeters, E., \& Malek, S.E., 2010, Science, 329, 1180

Cernicharo, J., Kahane, C., \& Guélin, M., 2000, A\&AS, 142, 181

Cernicharo, J., Heras, A.M., Tielens, A.G.G.M., et al., 2001a, ApJ, 546, L123

Cernicharo, J., Heras, A.M., Pardo, J.R., et al., 2001b, ApJ, 546, L127

Cernicharo, J., Goicoechea, J.R., Pardo, J.R., \& Asension-Ramos, A., 2006, ApJ, 642, 940

Charnley, S.B., 1997, ApJ, 481, 396

Comito, C., Schilke, P., Philips, T.G., et al., 2005, ApJS, 165, 127

Demyk, K., Mäder, H., Tercero, B., et al., 2007, A\&A, 466, 255

Fonfría, J.P., Cernicharo, J., Ritcher, M.J., \& Lacy, J.H., 2008, ApJ, 673, 445

Fonfría, J.P., Cernicharo, J., Ritcher, M.J., \& Lacy, J.H., 2008, ApJ, 729, 43 
Genzel, R., \& Stutzki, J., 1989, ARA\&A, 27, 41

Goldreich, P., \& Kwan, J., 1974, ApJ, 189, 441

Greaves, J.S., \& White, G.J., 1991, A\&ASS, 91, 237

Goicoechea, J.R., Rodríguez-Fernández, N.J., \& Cernicharo, J., 2004, ApJ, 600, 214

Herbig, G.H., 1995, ARA\&A, 33, 19

Herbst, E., \& van Dishoeck, E.F., 2009, ARA\&A, 47, 427

Herpin, F., Goicoechea, J.R., Pardo, J.R., \& Cernicharo, J., 2000, ApJ, 530, L129

Herpin, F., Goicoechea, J.R., Pardo, J.R., \& Cernicharo, J., 2002, ApJ, 577, 961

Jewell, P.R., Hollis, J.M., Lovas, F.J., \& Snyder, L.E., 1989, ApJS, 70, 833

Joblin, C., \& Tielens, A.G.G.M., 2011 (eds.), PAHs and the Universe: A Symposium to Celebrate the 25th Anniversary of the PAH Hypothesis, EAS Pub. Series, 46

Johansson, L.E.B., Andersson, C., Elldér, et al., 1984, A\&A, 130, 227

Lee, C.W., Cho, S.H., \& Lee, S.M., 2001, ApJ, 551, 333

Lee, C.W., \& Cho, S.H., 2002, Journal of The Korea Astronomical Society, 35, 187

Léger, A., \& Puget, J.L., 1984, A\&A, 137, L5

Lerate, M.R., Barlow, M.J., Swinyard, et al., 2006, MNRAS, 387, 1660

Marcelino, N., Cernicharo, J., Agúndez M., et al., 2007, ApJ, 665, L127

Marcelino, N., Cernicharo, J., Tercero, B., \& Roueff, E., 2009, ApJ, 690, L27

Margulès, L., Motiyenko, R., Demyk, K., Tercero, B., et al., 2009, A\&A, 493, 565

Margulès, L., Huet, T.R., Demaison, et al., 2010, ApJ, 714, 1120

Menten, K.M., Reid, M.J., Forbrich, J., \& Brunthaler, A., 2007, A\&A, 474, 515

Müller, H.S.P., Thorwirth, S., Roth, D.A., \& Winnewisser, G., 2001, A\&A, 370, L49

Müller, H.S.P., Schlöder, F., Stutzki, J., \& Winnewisser, G., 2005, J. Mol. Struct., 742, 215

Pickett, H.M., Poynter, R.L., Cohen, E.A., et al., 1998, JQSRT, 60, 883

Pilbratt, G.L., Riedinger, J.R., Passvogel, T., et al., 2010, A\&A, 518, L1

Schilke, P., Groesbeck, T.D., Blake, G.A., \& Philips, T.G., 1997, ApJS, 108, 301

Schilke, P., Benford, C.J., Hunter, T.R., et al., 2001, ApJS, 132, 281

Sellgren, K., Werner, M.W., Ingalls, J.G., et al., 2010, ApJ, 722, L54

Sutton, E.C., Blake, G.A., Masson, C.R., \& Philips, T.G., 1985, ApJS, 58, 341

Tercero, B., Cernicharo, J., Pardo, J.R., \& Goicoechea, J.R., 2010, A\&A, 517, 96

Tercero, B., Vincent L., Cernicharo, J., Viti S., \& Marcelino N., 2011, A\&A, 528, 26

Tercero, B., Margulès, L., Carvajal, et al., 2012, A\&A, 538, 119

Turner, B.E., 1989, ApJS, 70, 539

van Dishoeck, E.F., Kristensen, L.E., Benz, A., et al., 2011, PASP, 123, 138

White, G.J., Araki, M., Greaves, J.S., et al., 2003, A\&A, 407, 598

Ziurys, L.M., \& McGonagle, D., 1993 ApJS, 89, 155 